\title{
Semi-solid feeds may reduce the risk of aspiration pneumonia and shorten postoperative length of stay after percutaneous endoscopic gastrostomy (PEG)
}

Authors

Institutions
Ezekiel Wong Toh Yoon ${ }^{1}$, Kaori Yoneda² ${ }^{2}$ Kazuki Nishihara'

${ }^{1}$ Department of Internal Medicine (Gastroenterology), Hiroshima Kyoritsu Hospital, Hiroshima City, Japan

${ }^{2}$ Endoscopy Center, Hiroshima Kyoritsu Hospital, Hiroshima City, Japan submitted 5. May 2016 accepted after revision 20. August 2016

\section{Bibliography}

Dol http://dx.doi.org/ 10.1055/s-0042-117218 Published online: 15.11.2016 Endoscopy International Open 2016; 04: E1247-E1251

(c) Georg Thieme Verlag KG Stuttgart . New York E-ISSN 2196-9736

\section{Corresponding author}

Ezekiel Wong Toh Yoon, MD

Department of Internal

Medicine

Hiroshima Kyoritsu Hospital

2-20-20 Nakasu Asaminami-ku Hiroshima City

Japan

Phone: +81-82-879-1111

easybs@hotmail.com
Background and study aims: Feeding-related adverse events after percutaneous endoscopic gastrostomy (PEG) such as aspiration pneumonia may result in prolonged hospitalization and postoperative mortality. This study evaluated the efficacy of using semi-solid feeds to reduce feedingrelated adverse events and improve clinical outcomes.

Patients and methods: Patients who received PEG for enteral nutrition at our hospital between January 2014 and December 2015 were allocated to a postoperative feeding protocol that used either liquid feed or semi-solid feed. Baseline characteristics, postoperative feeding-related adverse events and clinical outcomes in the 2 groups were prospectively analysed and compared.

Results: One hundred and seventeen PEG patients (age range: 59-97 years, male: 53) were enrolled

\section{Introduction}

\section{$\nabla$}

Percutaneous endoscopic gastrostomy (PEG) is a minimally invasive procedure for long-term enteral tube feeding in patients with insufficient oral intake [1-3]. Although peristomal site infection is often noted as the most common adverse event (AE) after PEG tube placement, it is seldom life-threatening and considered a minor AE [4]. Feeding-related AEs have been identified as the main cause of death after PEG, with up to $50 \%$ of postoperative early mortality (30 days) being attributed to aspiration pneumonia $[5,6]$. This may be related to the persistence of gastroesophageal reflux (GER) of enteral feed after gastrostomy [7], even though PEG has been demonstrated to be superior to nasogastric tube feeding in terms of preventing GER $[8,9]$.

Recently, elemental diet (monomeric feed) has been shown to reduce the incidence of aspiration pneumonia [10]. However, limitations such as high cost, high osmolality, and almost zero lipid content may be an obstacle to the general use of with 72 patients given liquid feed and 45 patients receiving semi-solid feed. Baseline characteristics were similar in both groups. The semi-solid feed group experienced fewer incidence of feedingrelated aspiration pneumonia ( $2.2 \%$ vs. $22.2 \%, P<$ $0.005)$ and shorter postoperative hospital length of stay ( 12.7 days vs. 18.8 days, $P<0.01$ ). Significant differences were not observed in the frequency of peristomal infection $(11.1 \%$ vs. $12.5 \%$, $P=0.82)$, feeding-related diarrhea ( $2.2 \%$ vs. $12.5 \%$, $P=0.09)$ and 30 -day mortality rates ( $2.2 \%$ vs. $8.3 \%$, $P=0.25)$.

Conclusions: Semi-solid feeding may reduce the risk of aspiration pneumonia and shorten postoperative hospital length of stay after PEG. Semisolid feeds are safe to use and can be employed either as a first line feeding protocol or an alternative when liquid feeding is unsuccessful.

this specialized feed. It has been more than a decade since semi-solid feeds were developed as an alternative to conventional liquid feeds to prevent feeding-related AEs [11,12]. Unfortunately, there is limited published literature on this topic despite the wide usage of this feeding method in Japan. Amidst the growing popularity of this method and the introduction of National Healthcare Insurance coverage for a semi-solid feed prescription, we initiated a semi-solid feed protocol along with our existing post-PEG feeding protocols in 2014. In this study (NCT02858596), we prospectively evaluated the safety and efficacy of using semi-solid feed compared to conventional liquid feed using clinical outcomes of interests such as the development of feeding-related AEs, postoperative hospital length of stay and mortality. 


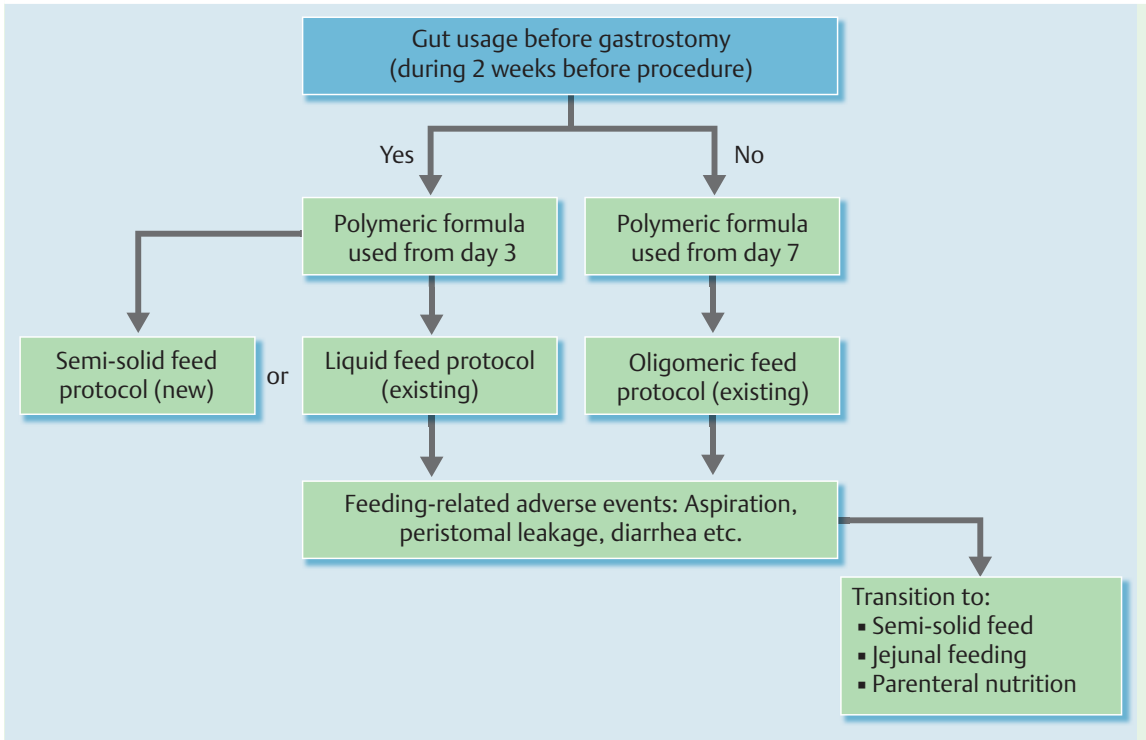

Fig. 1 Feeding protocols after percutaneous endoscopic gastrostomy (PEG) in Hiroshima Kyoritsu Hospital.

\section{Patients and methods \\ $\nabla$}

\section{Study design and patients}

Patients who received PEG for enteral nutrition at our hospital between January 2014 and December 2015 with the usage of gut (oral or nasogastric feeding) during the 2 weeks prior to procedure were allocated to receive a feeding protocol using either liquid feed or semi-solid feed. Gastrostomy tube used was $20 \mathrm{Fr}$ in size for all patients. Patient allocation depended on the attending physician ordering the procedure with most of the selection performed in a quasi-randomized manner. Patients with no recorded gut usage 2 weeks prior to PEG were given a slower feeding protocol starting with an oligomeric feed and not included in the study. This exclusion was to increase the homogeneity between the intervention (semi-solid feed) and control (liquid feed) groups in terms of baseline characteristics and protocol length. Likewise, procedure-related mortality cases $(<1 \%)$ were also excluded because the feeding protocol assigned could not be sufficiently evaluated. $\odot$ Fig. 1 summarizes the different postoperative feeding protocols used in our hospital.

The total amount and daily increment of tube feeding calories and water as well as the changes in parenteral nutrition used to supplement enteral feeding were the same for both the liquid feed and semi-solid feed groups. However, the increment in calories and water was stopped once daily requirements, as calculated by our nutrition support team, were reached. The characteristics and nutrient data of enteral feed used are shown in - Table 1, with the major difference being the dynamic viscosity of the feeds. Liquid feeds were administered using gravity-controlled infusion (averaging 100 to $200 \mathrm{~mL} / \mathrm{hr}$ ) from a ready-tohang bag whereas semi-solid feeds were injected directly into the stomach using a syringe via bolus infusion (5 to 10 minutes). For both groups, tube feeding was conducted intermittently according to meal times with gravity-controlled water administration (supplemented to match daily requirements) before infusion of feeds. After discharge, patients receiving semi-solid feed continue to receive the same type of prescription feed as currently covered by National Healthcare Insurance in Japan.

Data regarding baseline characteristics such as age, gender, comorbidities, and preoperative biomarkers (body mass index and blood laboratory markers collected on the day of procedure) as
Table 1 Characteristics and nutrient data of enteral feed used.

\begin{tabular}{|lcc|}
\hline Characteristics per $\mathbf{1 0 0}$ kcal & Liquid feed ${ }^{\mathbf{1}}$ & Semi-solid feed ${ }^{\mathbf{2}}$ \\
\hline Total weight, g & 100 & 66.7 \\
\hline Protein, g & 4 & 4 \\
\hline Fat, $\mathrm{g}$ & 2.8 & 2.2 \\
\hline Carbohydrate, g & 14.5 & 16.1 \\
\hline Dietary Fiber, g & 1 & 0.4 \\
\hline Water, ml & 84.5 & 44 \\
\hline Dynamic viscosity, mPa.s (or cP) & $5-10$ & 20,000 \\
\hline
\end{tabular}

${ }^{1}$ Meiji's Mei Balance 1.0 Z (Meiji Holdings Co.,Ltd., Tokyo, Japan)

${ }^{2}$ PG Soft (Terumo Corporation, Tokyo, Japan)

well as postoperative findings such as laboratory biomarker changes at day 7 after the procedure, AEs (feeding-related aspiration pneumonia, diarrhea etc.) and clinical outcomes of interest (postoperative length of stay and mortality) were compared for the 2 groups studied. Feeding-related aspiration pneumonia was diagnosed using clinical symptoms, with confirmation through radiologic findings or detection of enteral feed in the patient's trachea aspirate. Peristomal infection was assessed 1 week after the procedure using a previously validated scoring system (Jain's infection score) by assigning a score for erythema ( 0 to 4 ), induration ( 0 to 3 ) and exudate ( 0 to 4 ) [13]. Infection was defined as development of pus or a combined infection score of 8 or more. Diarrhea was evaluated using the King's Stool Chart and feedingrelated diarrhea was defined as an absolute daily fecal score of 15 or more after ruling out other causes such as drug-induced diarrhea or Clostridium difficile infection [14]. This study protocol was reviewed and approved by the ethics review committee of Hiroshima Kyoritsu Hospital. All patients, or their legal guardians, provided written informed consent for the procedures as well as enrollment in the study. The research was carried out in accordance with Japan's Ethical Guidelines for Epidemiological Research (2008) and the Declaration of Helsinki (2013).

\section{Statistical analysis}

Continuous variables are expressed as mean (standard deviation with range occasionally). Categorical variables are expressed as numbers (percentage). Comparisons for continuous variables were made using the Student $t$-test for normal data and the 


\begin{tabular}{|c|c|c|c|}
\hline & $\begin{array}{l}\text { Liquid feed } \\
(n=72)\end{array}$ & $\begin{array}{l}\text { Semi-solid feed } \\
(n=45)\end{array}$ & $P$ value \\
\hline Age, years, mean (SD, range) & $81.0(9.1,59-97)$ & $80.7(8.3,61-95)$ & 0.76 \\
\hline Gender (male/female) & $33 / 39$ & $20 / 25$ & 0.88 \\
\hline \multicolumn{4}{|l|}{ Comorbidities } \\
\hline Stroke, n (\%) & $53(73.6)$ & $36(80)$ & 0.43 \\
\hline Antithrombotic therapy, n (\%) & $26(36.1)$ & $17(37.8)$ & 0.86 \\
\hline Dementia, n (\%) & $29(40.3)$ & $13(28.9)$ & 0.21 \\
\hline Neurodegenerative disorders, n (\%) & $11(15.3)$ & $7(15.6)$ & 0.97 \\
\hline Respiratory disorders, n (\%) & $35(48.6)$ & $19(42.2)$ & 0.50 \\
\hline Diabetes mellitus, n (\%) & $16(22.2)$ & $14(31.1)$ & 0.28 \\
\hline Pressure ulcer, n (\%) & $17(23.6)$ & $8(17.8)$ & 0.45 \\
\hline Malignancy, n (\%) & $11(15.3)$ & $5(11.1)$ & 0.52 \\
\hline Partial gastric resection, $\mathrm{n}(\%)$ & $2(2.8)$ & $1(2.2)$ & 1.00 \\
\hline Hiatal hernia (> moderate size), n (\%) & $2(2.8)$ & $2(4.4)$ & 0.64 \\
\hline \multicolumn{4}{|l|}{ Preoperative biomarkers } \\
\hline Body mass index, $\mathrm{kg} / \mathrm{m}^{2}$, mean (SD) & $17.9(3.6)$ & $18.0(3.0)$ & 0.67 \\
\hline Hemoglobin, g/dL, mean (SD) & $11.6(1.8)$ & $11.3(2.0)$ & 0.52 \\
\hline Serum albumin, g/dL, mean (SD) & $2.8(0.5)$ & $2.9(0.6)$ & 0.34 \\
\hline $\mathrm{TLC}, / \mu \mathrm{L}$, mean $(\mathrm{SD})$ & $1508(863)$ & $1532(701)$ & 0.61 \\
\hline C-reactive protein, $\mathrm{mg} / \mathrm{dL}$, mean (SD) & $2.4(2.9)$ & $1.8(2.7)$ & 0.15 \\
\hline Total cholesterol, mg/dL, mean (SD) & $152(44)$ & $151(31)$ & 0.82 \\
\hline Blood urea nitrogen, mg/dL, mean (SD) & $23.7(17.8)$ & $22.8(14.0)$ & 0.75 \\
\hline PT-INR, mean (SD) & $1.20(0.23)$ & $1.13(0.11)$ & 0.07 \\
\hline
\end{tabular}

Table 2 Baseline characteristics of patients.

SD, standard deviation; TLC, total lymphocyte count; PT-INR, international normalized ratio of prothrombin time.

\begin{tabular}{|lccc|}
\hline & $\begin{array}{l}\text { Liquid feed } \\
(\mathbf{n = 7 2 )}\end{array}$ & $\begin{array}{l}\text { Semi-solid feed } \\
(\mathbf{n = 4 5 )}\end{array}$ & P value \\
\hline Biomarker changes at day 7 & & $-0.5(1.2)$ & 0.48 \\
\hline$\Delta$ Hemoglobin, g/dL, mean (SD) & $-0.6(0.9)$ & $0.4(3.2)$ & 0.79 \\
\hline$\Delta$ C-reactive protein, mg/dL, mean (SD) & $0.3(3.8)$ & $-0.1(0.4)$ & 0.61 \\
\hline$\Delta$ Serum albumin, g/dL, mean (SD) & $-0.1(0.3)$ & $0.6(7.8)$ & 0.95 \\
\hline$\Delta$ Blood urea nitrogen, mg/dL, mean (SD) & $-0.8(8.9)$ & & \\
\hline Adverse events & & $1(2.2)$ & $<0.005$ \\
\hline Feeding-related aspiration pneumonia & $16(22.2)$ & $5(11.1)$ & 0.82 \\
\hline Peristomal infection & $9(12.5)$ & $1.2(1.3)$ & 0.24 \\
\hline Jain's infection score, mean (SD) & $1.5(1.6)$ & $1(2.2)$ & 0.09 \\
\hline Feeding-related diarrhea & $9(12.5)$ & $0(0)$ & \\
\hline Tube obstruction & $0(0)$ & $12.7(7.5)$ & $<0.01$ \\
\hline Clinical outcomes & & $1(2.2)$ & 0.09 \\
\hline Length of stay, days, mean (SD) & $18.8(13.4)$ & $1(2.2)$ & 1.00 \\
\hline In-hospital mortality & $9(12.5)$ & $1(2.2)$ & 0.25 \\
\hline 14-day mortality & $3(4.2)$ & $2(4.4)$ & 0.20 \\
\hline 30-day mortality & $6(8.3)$ & $4(8.9)$ & 0.40 \\
\hline 60-day mortality & $9(12.5)$ & $0(0)$ & 0.05 \\
\hline 90-day mortality & $11(15.3)$ & $3(4.8)$ & \\
\hline 30-day readmission after discharge & & \\
\hline
\end{tabular}

Table 3 Postoperative clinical course of patients.

Values expressed as $\mathrm{n}(\%)$ unless otherwise specified. N/A: Not applicable.

Mann-Whitney U test for non-parametric data. Tests for proportionality between groups were made using the chi-square test (or Fisher's exact test when indicated). Logistic regression and analysis of covariance were used to evaluate possible factors that may be associated with the development of feeding-related aspiration pneumonia and postoperative length of stay. The number needed to treat (and 95\% confidence interval) for feeding-related pneumonia was also calculated. Statistical significance was defined as $P<0.05$ and analysis was performed using XLSTAT2014 for Windows (Addinsoft Ltd., Paris, France).

\section{Results}

In total, 117 patients (age range: 59-97 years, male: 53) were included in the study. Seventy-two patients were given liquid feed protocols while 45 patients received semi-solid feed protocols. Baseline characteristics of patients (age, gender, comorbidities and preoperative biomarkers) are listed in 0 Table2. There were no significant differences at baseline, including the frequency of comorbidities that may affect gastric motility such as previous gastric resection and hiatal hernia (moderate size and above) between the 2 groups. Indications for PEG were primarily dysphagia secondary to neurologic impairments or insufficient oral intake 
Table 4 Factors associated with postoperative length of stay (analysis of covariance).

\begin{tabular}{|c|c|c|}
\hline Variable & $\beta(95 \% \mathrm{Cl})^{1}$ & $P$ value \\
\hline Age & $0.09(-0.28$ to 0.10$)$ & 0.35 \\
\hline Male gender & $0.08(-0.12$ to 0.27$)$ & 0.43 \\
\hline Preoperative serum albumin & $0.02(-0.18$ to 0.22$)$ & 0.84 \\
\hline Preoperative C-reactive protein & $0.20(0.01$ to 0.40$)$ & $<0.05$ \\
\hline Semi-solid feed protocol & $-0.23(-0.41$ to -0.06$)$ & $<0.01$ \\
\hline
\end{tabular}

1 Standardized coefficients with $95 \%$ confidence interval using all variables listed in table. (Model's adjusted $R^{2}=0.13 ; F_{5,111}=3.21, P<0.01$ )

due to various underlying conditions as listed in the comorbidities section.

- Table 3 summarizes the postoperative clinical course of patients after PEG tube placement. Laboratory biomarkers were collected on postoperative day 7 and changes from preoperative values were compared between the 2 groups, with no significant differences found. Overall, feeding-related aspiration pneumonia was the most common $\mathrm{AE}$, followed by peristomal infection and feeding-related diarrhea. Compared to the Liquid feed group, the Semi-solid feed group had a significantly lower incidence of aspiration pneumonia ( $2.2 \%$ vs. $22.2 \%, P<0.005)$. Using logistic regression, after adjusting for preoperative C-reactive protein levels, patients in the Liquid feed group were more likely to develop feeding-related aspiration pneumonia compared to those in the Semi-solid feed group (Adjusted odds ratio 11.99, 95\% CI: 1.51 to $95.08, P<0.05$ ). After adjusting for preoperative serum albumin levels, the odds of developing aspiration pneumonia was also higher in the Liquid feed group (Adjusted odds ratio 12.51, $95 \%$ CI: 1.56 to $100.52, P<0.05)$. The calculated number needed to treat using semi-solid feed for feeding-related aspiration pneumonia was 5 patients ( $95 \% \mathrm{CI}: 3$ to 13 ). Although not statistically significant, there were fewer cases of feeding-related diarrhea in the Semi-solid feed group as well. The frequency of peristomal infection and the average infection scores were not significantly different between both groups. No tube obstruction was observed in either groups.

Postoperative length of stay was significantly shorter in the Semi-solid feed group (12.7 days vs. 18.8 days, $P<0.01$ ). There were also fewer deaths (in-hospital, 14-day, 30-day, 60-day and 90-day) in the Semi-solid feed group albeit not significantly different when compared to the Liquid feed group. After discharge, patients in the Semi-solid group also seemed less likely to be readmitted within the same month ( $0 \%$ vs. $4.8 \%, P=0.05)$. As shown in Table 4, analysis of covariance demonstrated that using semi-solid feed was associated with a shorter postoperative length of stay even after taking into account age, gender, preoperative serum albumin levels and preoperative $\mathrm{C}$-reactive protein levels ( $\beta-0.23,95 \% \mathrm{CI}$ : -0.41 to $-0.06, P<0.01)$.

\section{Discussion}

\section{$\nabla$}

PEG has been established as a safe and simple procedure for longterm enteral tube feeding. Nevertheless, feeding-related AEs such as aspiration pneumonia from GER of gastric feed may impede the successful use of PEG and contribute to postoperative mortality. Although jejunal feeding may reduce GER, an additional procedure is required and pump-assistance may also be necessary for enteral nutrition [15]. In Japan, the use of semi-solid feed in PEG patients to reduce feeding-related AEs such as aspiration pneumonia, peristomal leakage, and diarrhea is quite common. Semi-solid feed is considered to be more physiologic (swallowed food does not enter the stomach in the liquid form) and is reported in Japanese literature to reduce the incidence of GER, peristomal leakage, and diarrhea. Because it is given via bolus infusion, patients and care-givers are less restricted during feeding time, which may in turn contribute to a higher quality of life and lower incidence of pressure ulcers.

In an animal model of gastroesophageal reflux disease (GERD), use of semi-solid feed significantly reduced the frequency of GER during feeding and post-feeding periods [16]. In PEG patients, the efficacy of semi-solid feed in decreasing or inhibiting GER is not conclusive, with conflicting results being reported [17 - 19]. That may be due to differences in the dynamic viscosity of semi-solid feed used in various studies. A recent study also demonstrated that use of semi-solid feed was associated with fewer observational days with fever but the cause of fever in that study was not clearly defined [20].

This study showed that PEG patients with a postoperative feeding protocol using semi-solid feed had better clinical outcomes in terms of the frequency of feeding-related aspiration pneumonia and postoperative hospital length of stay. Fewer feeding-related AEs such as aspiration pneumonia in the Semi-solid feed group may have contributed to the shorter postoperative length of stay. Multivariate analysis also showed that using semi-solid feed had a positive effect on postoperative hospital length of stay, regardless of age, gender, and preoperative nutritional as well as inflammation status. Tube obstruction did not occur no matter which type of feed was used. The positive effect of using semi-solid feed also seemed to extend beyond discharge, as shown in the 30-day hospital readmission rates of both groups. Even with the reduction in aspiration pneumonia, significant improvement in mortality rates was not observed in the Semi-solid feed group. One possible explanation is that the attending physicians were free to change the type of feed when patients encountered feeding-related AEs. Further analysis revealed that 10 of 16 patients who had feeding-related aspiration pneumonia in the Liquid feed group changed to semi-solid feeding upon resuming enteral nutrition. Likewise, 4 of 9 patients who experienced feeding-related diarrhea in the Liquid feed group also had their feed changed to semi-solid type. This could be a reason why only postoperative length of stay (affected by AEs) was significantly different between the 2 groups.

Notable limitations of this study include the probability of selection bias (only quasi-randomized at best), which could explain why there were fewer patients enrolled in the Semi-solid group. The study also lacks long term follow-up data beyond 90 days (which is still in progress as an ongoing observational study). One potential disadvantage of using semi-solid feed is the requirement for additional water supplementation. As shown in - Table1, for the same amount of calories, semi-solid feed contains roughly half the water contained in a typical liquid feed. There is concern that administration of larger amounts of water may cause GER, resulting in aspiration. However, it has been demonstrated that gastric emptying is usually faster for water and liquid with lower caloric content [21]. 


\section{Conclusion}

\section{$\nabla$}

This study demonstrated that using semi-solid feeds may reduce the risk of aspiration pneumonia and shorten postoperative hospital length of stay after PEG. These clinical outcomes may lead to a better quality of life for patients and lower medical costs. Largescale randomized controlled trials are warranted to confirm this. Semi-solid feeds are safe to use and can be employed either as a first-line feeding protocol or an alternative when liquid feeding is unsuccessful.

\section{Competing interests: None}

\section{References}

1 Gauderer MW, Ponsky JL, Izant RJ Jr. Gastrostomy without laparotomy: a percutaneous endoscopic technique. J Pediatr Surg 1980; 15: 872 875

2 DeLegge MH. Percutaneous endoscopic gastrostomy. Am J Gastroenterol 2007; 102: 2620-2623

3 Suzuki Y, Tamez S, Murakami A et al. Survival of geriatric patients after percutaneous endoscopic gastrostomy in Japan. World J Gastroenterol 2010; 16: 5084-5091

4 Richter-Schrag HJ, Richter S, Ruthmann $O$ et al. Risk factors and complications following percutaneous endoscopic gastrostomy: a case series of 1041 patients. Can J Gastroenterol 2011; 25: 201 - 206

5 Laskaratos FM, Walker M, Walker $M$ et al. Predictive factors for early mortality after percutaneous endoscopic and radiologically-inserted gastrostomy. Dig Dis Sci 2013; 58: 3558 - 3565

6 Azzopardi N, Ellul P. Pneumonia and mortality after percutaneous endoscopic gastrostomy insertion. Turk J Gastroenterol 2013; 24: $109-116$

7 Nishiwaki S, Araki H, Goto $\mathrm{N}$ et al. Clinical analysis of gastroesophageal reflux after PEG. Gastrointest Endosc 2006; 64: 890-896

8 Jung SH, Dong SH, Lee JY et al. Percutaneous Endoscopic Gastrostomy Prevents Gastroesophageal Reflux in Patients with Nasogastric Tube Feeding: A Prospective Study with 24-Hour pH Monitoring. Gut Liver 2011; 5: $288-292$

9 Lee TH, Shiun YC. Changes in gastroesophageal reflux in patients with nasogastric tube followed by percutaneous endoscopic gastrostomy. J Formos Med Assoc 2011; 110: 115-119
10 Horiuchi A, Nakayama $Y$, Sakai $R$ et al. Elemental diets may reduce the risk of aspiration pneumonia in bedridden gastrostomy-fed patients. Am J Gastroenterol 2013; 108: 804-810

11 Kanie J, Suzuki Y, Iguchi A et al. Prevention of gastroesophageal reflux using an application of half-solid nutrients in patients with percutaneous endoscopic gastrostomy feeding. J Am Geriatr Soc 2004; 52: $466-467$

12 Kanie J, Suzuki Y, Akatsu $H$ et al. Prevention of late complications by half-solid enteral nutrients in percutaneous endoscopic gastrostomy tube feeding. Gerontology 2004; 50: 417-419

13 Jain NK, Larson DE, Schroeder KW et al. Antibiotic prophylaxis for percutaneous endoscopic gastrostomy. A prospective, randomized, double-blind clinical trial. Ann Intern Med 1987; 107: 824-828

14 Whelan K, Judd PA, Taylor MA. Assessment of fecal output in patients receiving enteral tube feeding: validation of a novel chart. Eur J Clin Nutr 2004; 58: 1030-1037

15 Adams GF, Guest DP, Ciraulo DL et al. Maximizing tolerance of enteral nutrition in severely injured trauma patients: a comparison of enteral feedings by means of percutaneous endoscopic gastrostomy versus percutaneous endoscopic gastrojejunostomy. J Trauma 2000; 48: 459-464

16 Tanishima Y, Fujita T, Suzuki Y et al. Effects of half-solid nutrients on gastroesophageal reflux in beagle dogs with or without cardioplasty and intrathoracic cardiopexy. J Surg Res 2010; 161: 272 - 277

17 Nishiwaki S, Araki H, Shirakami Y et al. Inhibition of gastroesophageal reflux by semi-solid nutrients in patients with percutaneous endoscopic gastrostomy. JPEN J Parenter Enteral Nutr 2009; 33: 513 - 519

18 Shimizu A, Muramatsu H, Kura T et al. Incidence of gastroesophageal reflux associated with percutaneous endoscopic gastrostomy contrast agent viscosity: a randomized controlled crossover trial. Eur J Clin Nutr 2016: [Epub ahead of print] DOI 10.1038/ejcn.2016.76

19 Adachi K, Furuta K, Morita T et al. Half-solidification of nutrient does not decrease gastro-esophageal reflux events in patients fed via percutaneous endoscopic gastrostomy. Clin Nutr 2009; 28: 648-651

20 Shizuku T, Adachi K, Furuta K et al. Efficacy of half-solid nutrient for the elderly patients with percutaneous endoscopic gastrostomy. J Clin Biochem Nutr 2011; 48: 226-229

21 Okabe T, Terashima H, Sakamoto A. Determinants of liquid gastric emptying: comparisons between milk and isocalorically adjusted clear fluids. Br J Anaesth 2015; 114: 77-82 